\title{
LWS-Based Time-Domain Synthetic Algorithm with Constant Amplitude in Radar Transmit Waveform
}

\author{
Bin Wang $\mathbb{D}^{1}$ Shumin $\mathrm{Li},{ }^{2}$ and Fengming Xin $^{2}$ \\ ${ }^{1}$ School of Electrical and Electronic Engineering, Shijiazhuang Tiedao University, Shijiazhuang, China \\ ${ }^{2}$ School of Computer and Communication Engineering, Northeastern University at Qinhuangdao, Qinhuangdao, China \\ Correspondence should be addressed to Bin Wang; wangbinneu@qq.com
}

Received 4 April 2020; Revised 22 June 2020; Accepted 29 June 2020; Published 17 July 2020

Academic Editor: Juan C. Cano

Copyright (c) 2020 Bin Wang et al. This is an open access article distributed under the Creative Commons Attribution License, which permits unrestricted use, distribution, and reproduction in any medium, provided the original work is properly cited.

As the working electromagnetic environment of radar is becoming more and more complex, the research on single-target problem can no longer meet the actual needs. Therefore, this paper changes single-target problem into multitarget problem based on the traditional water-filling method. In order to better meet the actual environment and requirement, this paper proposes a new algorithm with phase factor added based on the general water-filling algorithm. A time-domain synthetic algorithm based on the linear weighted summation (LWS) method is proposed. The minimum mean square error (MMSE) is used to measure the proximity between the radar multitarget optimal transmit waveform algorithm and the time-domain synthetic algorithm. The MMSE-based cost function is a nonlinear least square error estimation problem, and the time-domain waveform can be obtained after solving it. Simulation results show that the energy spectrum density (ESD) of the synthetic signal after adding the phase is very close to the optimal energy spectrum density of LWS algorithm in three cases. The time-domain synthetic algorithm based on signal-to-interference-plus-noise ratio (SINR) has better detection and recognition performance than that based on mutual information (MI).

\section{Introduction}

Traditional radar usually combines fixed transmit signals with receiving end and uses adaptive technology and filtering algorithms to perform calculation process to complete a series of detection tasks [1,2]. The measurement and resolution of targets based on radar and target detection are theoretically determined by the transmit waveform. Therefore, under the circumstance that the radar is relatively complicated, the clutter is relatively concentrated. In the case of multiple targets, if the working environment changes, it is difficult to achieve the desired effect by only relying on the adaptive processing of the receiving end. In [3], Fan proposes that when multiple targets are angularly separable, i.e., when the targets are in different radar beams, the multitarget waveform design problem is relatively simple, and the radar can search, detect, and identify the target through multiple scans. In [4, 5], Romero proposes that the relationship between deterministic targets and random targets is determined by mutual information and signal-to-interference ratio. In [6], Wei establishes a limited-bandwidth radar system, and an alternate iterative algorithm is proposed to optimize the phase modulation baseband waveform by maximizing the signal-to-noise ratio of the receiver filter output. In this method, phase modulation waveform can gain more target information and improve extended target detection performance. Gong et al. propose that phase modulation can minimize the SCNR loss by minimizing the mean square spectrum difference between the optimal waveforms, and the algorithm can improve the overall detection performance of the radar system [7-9].

Cognitive radar system is a combination of prior knowledge and surrounding environmental factor. It perceives the environment through interactive learning and adjusts the transmitter to adapt to the working condition. In 2013 and 2014, Wang at al. [10, 11] proposed the waveform optimization method for single extended target recognition. Every received echo is processed to obtain a priori 
information for the next transmit waveform of each target and meanwhile provides guidance for the next transmission. In [12], Jiu et al. reduce the uncertainty of the target characteristics by maximizing the mutual information between the target echo and the extended target characteristics. This idea is then applied to the problem of waveform optimization in target recognition to increase the discrimination of various targets. In [13], Dong derives the global optimal solution of the transmit signal ESD according to the maximum SCNR criterion. In order to obtain a meaningful time-domain signal, phase modulation technology is used [14]. This method combines the minimum mean square error (MMSE) with an iterative algorithm to optimize the ESD and synthesizes a constant-amplitude time-domain signal that meets the requirements of radar transmission. In [15], Zhu et al. propose a method to solve multitarget classification problems, and simulation results show that the optimal waveform-based sequential testing can be decided through the reduction of the average illumination number. Furthermore, the conclusion indicates a significant improvement over the nonoptimal waveforms with unknown clutter parameters.

In cognitive radar system, a closed-loop feedback is formed by the transmitter, environment, and receiver. Then, the transmit waveform can be optimized to improve the radar estimation performance. In 2016, Peng and $\mathrm{Wu}$ [16] proposed a Kalman filtering- (KF-) based method to exploit the temporal correlation of target scattering coefficients (TSCs) and improve the corresponding estimation performance. In 2017, Yue et al. [17] considered the constantmodulus (CM) waveform optimization problem to improve high-resolution range (HRR) profiling performance for stationary targets. Simulation results show that the optimized CM waveforms can dramatically enhance the profiling performance. In [18], the information-theoretic waveform for target classification based on spectral variance is studied, and its advantages have been shown in simulations. The problem of radar constant-modulus (CM) waveform design for the detection of multiple targets was considered by yue et al. in 2018 [19]. This paper shows that the designed CM waveforms perform satisfactorily, even when compared with their counterparts without constraints on the peak-to-average ratio. In [20], Wang et al. research the multitarget detection problem and propose an adaptive waveform design algorithm for cognitive MIMO radar. In this method, the multitarget detection is modeled as a multihypothesis testing. The multihypothesis testing is investigated according to the sequentially received data. In 2019, Hao et al. researched the waveform design problem in cognitive radar for extended target estimation in the presence of signal-dependent clutter, with the constraint of peakto-average power ratio (PAR) [21]. Other relevant research can be referred to in [22-24].

According to the detailed analysis of the above various research results, we can know that the research and design of the multitarget radar transmit waveform have not been completely resolved and do not meet the actual needs well. This paper is to study and design the multitarget radar waveform on the basis of information theory. Based on the general water-filling algorithm, the phase information of the multitarget radar transmit waveform is considered, so that more target information can be obtained accurately, and the actual application demand can be better met. We mainly adopt the idea of constant envelope to synthesize the optimal transmit waveform in time domain and finally determine the phase information of multiple targets. Finally, the mean square error is used to qualitatively compare the multitarget optimal transmit waveform with the time-domain synthetic waveform. It can be known that the time-domain synthetic signal is very close to the optimal transmit waveform, and more target information can be obtained to achieve the purpose of our research.

In the second section, the target model based on information theory from single target to multiple targets is established. The third section gives the detailed solution of the three cases based on the LWS algorithm on the basis of the water-filling method. The fourth section proposes the synthesis of the time-domain waveform for these three cases. Then, the time-domain signal is obtained by cyclic iteration. The fifth section verifies the feasibility of the time-domain synthetic signal method in the three cases through simulation experiments. In the sixth section, the conclusion is given.

\section{Problem Formulation}

2.1. Single-Target Radar Transmit Waveform Model. Figure 1 is single-target radar transmit waveform model. $x(t)$ is transmit waveform, $y(t)$ is receive waveform, $h(t)$ is a known target response of the radar transmit information, $n(t)$ is an additive white Gaussian noise, and $c(t)$ is clutter. After the corresponding Fourier transform, the frequency domain expressions are, respectively, $X(f), Y(f), H(f)$, $S_{n n}(f)$, and $S_{c c}(f)$. The entire process bandwidth is $B$ and the duration is $T_{y}$. The expression of single-target energy spectral density is shown in

$$
\sigma_{h}^{2}(f)=E\left[\left|H(f)-\mu_{h}(f)\right|^{2}\right] .
$$

Form the knowledge of information theory, we can obtain the objective function of single target and constraints based on mutual information and signal-to-interferenceplus-noise ratio. It can be concluded in

$$
\begin{array}{ll}
\max _{M I} & T_{y} \int_{B} \ln \left[1+\frac{|X(f)|^{2} \sigma_{h}^{2}(f)}{T_{y}\left\{S_{n n}(f)+|X(f)|^{2} S_{c c}(f)\right\}}\right] \mathrm{d} f \\
\text { s.t. } & \int_{B}|X(f)|^{2} \mathrm{~d} f \leq E_{x}, \\
& \max _{\text {SINR }} \quad T_{y} \int_{B} \frac{|X(f)|^{2} \sigma_{h}^{2}(f)}{T_{y}\left\{S_{n n}(f)+|X(f)|^{2} S_{c c}(f)\right\}} \mathrm{d} f \\
& \text { s.t. } \quad \int_{B}|X(f)|^{2} \mathrm{~d} f \leq E_{x},
\end{array}
$$

2.2. Multitarget Radar Transmit Waveform Model. Figure 2 shows the multitarget radar transmit waveform model. The corresponding parameters are the same as those 


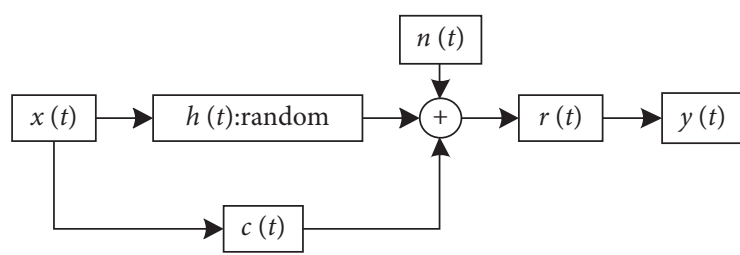

Figure 1: Single-target radar transmit waveform model.

of the single-target model. The difference is that as the single target expands to multiple targets, the target energy spectrum variance $\sigma_{h_{l}}^{2}(f)$ changes. According to multitarget radar waveform design model of Goodman, it can be known that the variance of the energy spectrum $\sigma_{h_{1}}^{2}(f)$ of each target will vary with the target, $\sigma_{h_{l}}^{2}(f)=E\left[\mid H_{l}(f)\right.$ $\left.\left.\mu_{h_{l}}(f)\right|^{2}\right]$. We suppose that multiple targets are transmitted in the same beam. It is convenient to study all targets as a whole large target, and the energy spectrum variance is $\sigma_{h}^{2}(f)$. However, there is a problem in the research process. In the designing process, the optimal transmit waveform under a certain target state is not necessarily optimal for the state of other targets at the same time. Therefore, aiming at this problem, this paper proposes the idea of weight distribution. Each independent target is assigned by its corresponding weight. For the parameter of distance, more weight is assigned to targets that are closer to the transmit end; otherwise, less weight is assigned. This method can ensure that each target is relatively optimal in the process of studying the optimal transmit waveform and make the research more reasonable and feasible. This treatment will also solve the problem of differences between targets, so we propose a weighted energy spectrum variance, $\sigma_{h}^{2}(f)=\sum_{l=1}^{L}$ $w_{l} \sigma_{h_{l}}^{2}(f)$, and $\sum_{l=1}^{L} w_{l}=1$, where $w_{l}=\left|\alpha_{l}\right|^{2}$.

This paper proposes the LWS idea. This method assigns corresponding weights to each target reasonably, combines the previous analysis, and finally determines the objective function and constraints of the multitarget radar transmit waveform design. It can be expressed as

$$
\begin{aligned}
& \max _{M I} T_{y} \int_{B} \ln \left[1+\frac{|X(f)|^{2} \sum_{l=1}^{L} w_{l} \sigma_{h_{l}}^{2}(f)}{T_{y}\left\{S_{n n}(f)+|X(f)|^{2} S_{c c}(f)\right\}}\right] \mathrm{d} f \\
& \text { s.t. } \quad \int_{B}|X(f)|^{2} \mathrm{~d} f \leq E_{x} \text {, } \\
& \max _{\operatorname{SINR}} \quad T_{y} \int_{B} \frac{|X(f)|^{2} \sum_{l=1}^{L} w_{l} \sigma_{h_{l}}^{2}(f)}{T_{y}\left\{S_{n n}(f)+|X(f)|^{2} S_{c c}(f)\right\}} \mathrm{d} f \\
& \text { s.t. } \quad \int_{B}|X(f)|^{2} \mathrm{~d} f \leq E_{x} \text {. }
\end{aligned}
$$

\section{Linear Weighted Sum (LWS) Algorithm}

\subsection{MI-Based Stochastic Multitarget Radar Waveform Design}

3.1.1. MI-Based Waveform Design for Stochastic Multitarget in Noise Only. Suppose that there are $L$ targets within radar range, and the targets are separable in distance. Based on the information theory of multitarget radar transmit waveform,

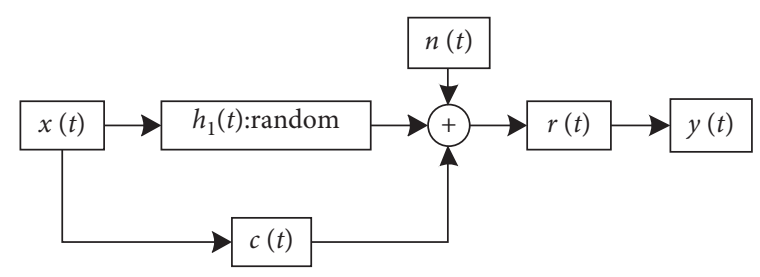

Figure 2: Multitarget radar transmit waveform model.

we further identify and determine more target information. For each target, they are all independently distributed. They all obey a Gaussian random process with a mean of 0 and a variance of $\sigma_{h_{l}}^{2}(f)$. Energy spectral density of noise is $S_{n n}(f)$ with the value 1 during the research process. Based on the mutual information research, there are two cases: clutter and no clutter. $S_{c c}(f)$ represents the energy spectral density of clutter (the optimal transmit waveform energy spectrum in a dense Gaussian distributed clutter environment), and $w_{l}$ is the corresponding weight of each target. A general relationship between the corresponding weights is $\sum_{l=1}^{L} w_{l}=1$.

The paper expands a single-target situation to a multitarget situation, and the targets are distinguishable in distance. This is useful for target recognition and will not cause confusion of information between targets. Based on the traditional waterfilling algorithm, the main constraints are the energy constraint and bandwidth constraint in the finite time domain. Through the above analysis, the objective function based on the LWS algorithm under energy and bandwidth constraints in the presence of noise only is finally determined as

$$
\max T_{y} \int_{B} \ln \left[1+\frac{|X(f)|^{2} \sum_{l=1}^{L} w_{l} \sigma_{h_{l}}^{2}(f)}{T_{y}\left\{S_{n n}(f)\right\}}\right] \mathrm{d} f
$$

$$
\text { s.t. } \quad \int_{B}|X(f)|^{2} \mathrm{~d} f \leq E_{x} \text {. }
$$

The multitarget function is a concave function through verification, and using the Lagrange multiplier method, we can obtain

$$
\begin{aligned}
L\left(|X(f)|^{2}, \lambda\right)= & T_{y} \int_{B} \ln \left[1+\frac{|X(f)|^{2} \sum_{l=1}^{L} w_{l} \sigma_{h_{l}}^{2}(f)}{T_{y}\left\{S_{n n}(f)\right\}}\right] \mathrm{d} f \\
& -\lambda\left[\int_{B}|X(f)|^{2} \mathrm{~d} f-E_{x}\right] .
\end{aligned}
$$

By solving formula (7), we can know that

$$
\begin{aligned}
\frac{\partial L\left(|X(f)|^{2}, \lambda\right)}{\partial|X(f)|^{2}}= & T_{y} \cdot \ln \left[1+\frac{|X(f)|^{2} \sum_{l=1}^{L} w_{l} \sigma_{h_{l}}^{2}(f)}{T_{y}\left\{S_{n n}(f)\right\}}\right] \\
& -\lambda|X(f)|^{2},
\end{aligned}
$$

$$
\frac{\partial^{2} L\left(|X(f)|^{2}, \lambda\right)}{\partial\left[|X(f)|^{2}\right]^{2}}=\frac{T_{y} \cdot \sum_{l=1}^{L} w_{l} \sigma_{h_{l}}^{2}(f)}{T_{y} S_{n n}(f)+|X(f)|^{2} \sum_{l=1}^{L} w_{l} \sigma_{h_{l}}^{2}(f)}-\lambda .
$$

Let $\partial^{2} L\left(|X(f)|^{2}, \lambda\right) / \partial\left[|X(f)|^{2}\right]^{2}=0$, and the specific expression of $|X(f)|^{2}$ is 


$$
|X(f)|^{2}=\max \left(0, \frac{T_{y}}{\lambda}-\frac{S_{n n}(f) T_{y}}{\sum_{l=1}^{L} w_{l} \sigma_{h_{l}}^{2}(f)}\right) .
$$

Through calculation and analysis, it is known that, under conditions of energy constraint and bandwidth constraint, the Lagrange multiplier $\lambda$ searches within $0 \leq \lambda \leq \max$ $\left[\sum_{l=1}^{L} w_{l} \sigma_{h_{l}}^{2}(f) / S_{n n}(f)\right]$, which can make the transmit waveform meet the constant energy value of signal $x(t)$. Therefore, the optimal transmit waveform expression in this case can be shown as

$$
\varepsilon_{\mathrm{opt}}(f)=\frac{T_{y}}{\lambda}-\frac{S_{n n}(f) T_{y}}{\sum_{l=1}^{L} w_{l} \sigma_{h_{l}}^{2}(f)} .
$$

3.1.2. MI-Based Waveform Design for Stochastic Multitarget in Signal-Dependent Interference. Under the premise of a multitarget recognition algorithm based on mutual information in the case of noise only in Section 3.1.1, a multitarget recognition algorithm based on mutual information in the presence of clutter is proposed.

According to the distance-sensitive principle proposed by Wang et al. [11], each target is distributed independently of each other without causing confusion. Based on the general water-filling algorithm, energy constraint and bandwidth constraint are the conditions involved in the process. Through the above analysis, the objective function based on energy and bandwidth constraints in the presence of signal-dependent interference is finally determined as

$$
\begin{array}{ll}
\max & T_{y} \int_{B} \ln \left[1+\frac{|X(f)|^{2} \sum_{l=1}^{L} w_{l} \sigma_{h_{l}}^{2}(f)}{T_{y}\left\{S_{n n}(f)+|X(f)|^{2} S_{c c}(f)\right\}}\right] \mathrm{d} f \\
\text { s.t. } & \int_{B}|X(f)|^{2} \mathrm{~d} f \leq E_{x} .
\end{array}
$$

As multitarget function is a concave function, the Lagrange multiplier method is used to solve this problem:

$$
\begin{aligned}
L\left(|X(f)|^{2}, \lambda\right)= & T_{y} \int_{B} \ln \left[1+\frac{|X(f)|^{2} \sum_{l=1}^{L} w_{l} \sigma_{h_{l}}^{2}(f)}{T_{y}\left\{S_{n n}(f)+|X(f)|^{2} S_{c c}(f)\right\}}\right] \mathrm{d} f \\
& -\lambda\left[\int_{B}|X(f)|^{2} \mathrm{~d} f-E_{x}\right],
\end{aligned}
$$

then, we can know that

$$
\begin{aligned}
& \frac{\partial L\left(|X(f)|^{2}, \lambda\right)}{\partial|X(f)|^{2}}=T_{y} \cdot \ln \left[1+\frac{|X(f)|^{2} \sum_{l=1}^{L} w_{l} \sigma_{h_{l}}^{2}(f)}{T_{y}\left\{S_{n n}(f)+|X(f)|^{2} S_{c c}(f)\right\}}\right]-\lambda|X(f)|^{2}, \\
& \frac{\partial^{2} L\left(|X(f)|^{2}, \lambda\right)}{\partial\left[|X(f)|^{2}\right]^{2}}=\frac{S_{n n}(f) \cdot \sum_{l=1}^{L} w_{l} \sigma_{h_{l}}^{2}(f)}{\left[T_{y}\left(S_{n n}(f)+|X(f)|^{2} S_{c c}(f)\right)+|X(f)|^{2} \sum_{l=1}^{L} w_{l} \sigma_{h_{l}}^{2}(f)\right]\left[S_{n n}(f)+|X(f)|^{2} S_{c c}(f)\right]}-\frac{\lambda}{T_{y}} .
\end{aligned}
$$

Assume $\partial^{2} L\left(|X(f)|^{2}, \lambda\right) / \partial\left[|X(f)|^{2}\right]^{2}=0$, and use the root finding formula to find the specific expression of $|X(f)|^{2}$. We can obtain that

$$
|X(f)|^{2}=\frac{-B(f) \pm \sqrt{B^{2}(f)-4 A(f) C(f)}}{2 A(f)} .
$$

As the expression of energy spectrum is always positive, so it can be expressed as

$$
\begin{gathered}
|X(f)|^{2}=\frac{-B(f)+\sqrt{B^{2}(f)-4 A(f) C(f)}}{2 A(f)} \\
A(f)=T_{y}\left[S_{c c}^{2}(f)+S_{c c}(f) \cdot \sum_{l=1}^{L} w_{l} \sigma_{h_{l}}^{2}(f)\right] \\
B(f)=2 T_{y} \cdot S_{n n}(f) \cdot S_{c c}(f)+T_{y} \cdot S_{n n}(f) \cdot \sum_{l=1}^{L} w_{l} \sigma_{h_{l}}^{2}(f)
\end{gathered}
$$

$$
C(f)=T_{y} \cdot S_{n n}^{2}(f)-\frac{T_{y}}{\lambda} \cdot S_{n n}(f) \cdot \sum_{l=1}^{L} w_{l} \sigma_{h_{l}}^{2}(f) .
$$

Assume $T_{y} / \lambda=A$, and simplification is performed on the above formulae (18)-(20). The final transmit waveform can be expressed as

$$
\max |X(f)|^{2}=\max \left(-R(f)+\sqrt{R^{2}(f)+S(f)(A(f)-D(f))}, 0\right),
$$

$$
\begin{aligned}
& R(f)=\frac{2 S_{c c}^{2}(f)+T_{y} \cdot S_{n n}(f) \cdot \sum_{l=1}^{L} w_{l} \sigma_{h_{l}}^{2}(f)}{S_{c c}^{4}(f)+S_{c c}^{2}(f) \cdot \sum_{l=1}^{L} w_{l} \sigma_{h_{l}}^{2}(f)}, \\
& S(f)=\frac{S_{n n}(f) \cdot T_{y} \cdot \sum_{l=1}^{L} w_{l} \sigma_{h_{l}}^{2}(f)}{S_{c c}^{4}(f)+S_{c c}^{2}(f) \cdot \sum_{l=1}^{L} w_{l} \sigma_{h_{l}}^{2}(f)}, \\
& D(f)=\frac{S_{n n}(f) \cdot T_{y}}{\sum_{l=1}^{L} w_{l} \sigma_{h_{l}}^{2}(f)},
\end{aligned}
$$


where the Lagrange multiplier is determined by the energy constraint of radar transmit waveform. It can be seen that the optimal transmit waveform energy allocation is mainly based on the distribution of the extended target impulse response, clutter, and noise. Although energy spectral density (ESD) of the optimal transmit waveform $\varepsilon_{\text {opt }}(f)$ is obtained, the ESD of the optimal transmit signal $x(t)$ lacks phase information at the same time. Therefore, it is necessary to further synthesize a time-domain signal of constant amplitude and limited time-width based on the ESD of the optimal transmit waveform.

In the end, the expression of the optimal transmit waveform in signal-dependent interference can be expressed as

$$
\varepsilon_{\text {opt }}(f)=-R(f)+\sqrt{R^{2}(f)+S(f)(A(f)-D(f))} .
$$

\subsection{SINR-Based Stochastic Multitarget Radar Waveform} Design in Signal-Dependent Interference. The above two cases are based on MI, and the third case is based on SINR with changing criterion conditions. We consider the multitarget radar waveform design problem based on the signalto-interference ratio in the presence of signal-dependent interference. At this time, there are still $L$ targets within radar range, where $S_{n n}(f)$ represents the energy spectral density of noise. Let $S_{n n}(f)=1$, and $S_{c c}(f)$ represents the energy spectral density of clutter (the optimal transmit waveform energy spectrum in a dense Gaussian distribution clutter environment). The corresponding target weight distribution satisfies the relationship $\sum_{l=1}^{L} w_{l}=1$. According to the analysis, the objective function based on the signal-tointerference ratio is finally determined as follows:

$$
\max T_{y} \int_{B} \frac{|X(f)|^{2} \sum_{l=1}^{L} w_{l} \sigma_{h_{l}}^{2}(f)}{T_{y}\left\{S_{n n}(f)+|X(f)|^{2} S_{c c}(f)\right\}} \mathrm{d} f
$$

$$
\text { s.t. } \quad \int_{B}|X(f)|^{2} \mathrm{~d} f \leq E_{x} \text {. }
$$

At this time, the objective function is also a concave function. Use the Lagrange multiplier method and assume that the multiplier is $\lambda$. Then, we can obtain

$$
\begin{aligned}
L\left(|X(f)|^{2}, \lambda\right)= & T_{y} \int_{B} \frac{|X(f)|^{2} \sum_{l=1}^{L}\left\{w_{l} \sigma_{h_{l}}^{2}(f)+|X(f)|^{2} S_{c c}(f)\right\}}{d f} \\
& -\lambda\left[\int_{B}|X(f)|^{2} \mathrm{~d} f-E_{x}\right] .
\end{aligned}
$$

It can be known from the calculation that

$$
\begin{aligned}
& \frac{\partial L\left(|X(f)|^{2}, \lambda\right)}{\partial|X(f)|^{2}}=T_{y} \cdot \frac{|X(f)|^{2} \sum_{l=1}^{L} w_{l} \sigma_{h_{l}}^{2}(f)}{T_{y}\left\{S_{n n}(f)+|X(f)|^{2} S_{c c}(f)\right\}}-\lambda|X(f)|^{2} \\
& \frac{\partial^{2} L\left(|X(f)|^{2}, \lambda\right)}{\partial\left[|X(f)|^{2}\right]^{2}}=\frac{S_{n n}(f) \cdot \sum_{l=1}^{L} w_{l} \sigma_{h_{l}}^{2}(f)}{\left[S_{n n}(f)+|X(f)|^{2} S_{c c}(f)\right]^{2}}-\lambda
\end{aligned}
$$

Let $\partial^{2} L\left(|X(f)|^{2}, \lambda\right) / \partial\left[|X(f)|^{2}\right]^{2}=0$, and solve the specific expression of $|X(f)|^{2}$ as

$$
|X(f)|^{2}=\frac{\sqrt{\sum_{l=1}^{L} w_{l} \sigma_{h_{l}}^{2}(f) \cdot S_{n n}(f) / \lambda}-S_{n n}(f)}{S_{c c}(f)} .
$$

As the energy spectrum is always positive, the expression of $|X(f)|^{2}$ is shown as

$$
|X(f)|^{2}=\max \left[\frac{\sqrt{\sum_{l=1}^{L} w_{l} \sigma_{h_{l}}^{2}(f) \cdot S_{n n}(f) / \lambda}-S_{n n}(f)}{S_{c c}(f)}, 0\right] .
$$

Substitute the optimal radar transmit signal into the energy constraint condition to solve the Lagrange multiplier $\lambda$, and we can obtain that

$$
|X(f)|^{2}=\int_{B} \max \left[\frac{\sqrt{\sum_{l=1}^{L} w_{l} \sigma_{h_{l}}^{2}(f) \cdot S_{n n}(f) / \lambda}-S_{n n}(f)}{S_{c c}(f)}, 0\right] \mathrm{d} f .
$$

Under the energy constraint, the Lagrange multiplier searches within the range $0 \leq \lambda \leq \max \left[\sum_{l=1}^{L} w_{l} \sigma_{h_{l}}^{2}(f) / S_{n n}(f)\right]$ to make it meet the constant energy value of the signal $x(t)$.

The final energy spectrum density of the optimal transmit waveform is as follows:

$$
\varepsilon_{\mathrm{opt}}(f)=\max \left[\frac{\sqrt{\sum_{l=1}^{L} w_{l} \sigma_{h_{l}}^{2}(f) \cdot S_{n n}(f) / \lambda}-S_{n n}(f)}{S_{c c}(f)}, 0\right],
$$

where the Lagrange multiplier $\lambda$ is determined by the constraint of the radar transmit waveform energy. It can be seen that the energy allocation of the optimal waveform is mainly allocated according to the magnitude of the extended target impulse response, clutter, and noise. Although the ESD of the optimal transmit waveform $\varepsilon_{\text {opt }}(f)$ is obtained, the ESD of the optimal transmit signal also lacks phase information at this time.

\section{Time-Domain Waveform Synthesis and Computational Complexity Analysis}

4.1. Time-Domain Waveform Synthesis. The radar transmit signal determined by the antenna structure and nonlinear amplifier in modern radar systems must be constant amplitude. Assume that the radar transmit signal is a form of nonlinear frequency modulation, and its amplitude is $c$ with value 1 . The expression is as follows:

$$
x(t)=c e^{j \varphi(t)}, \quad t \in\left[-\frac{T}{2}, \frac{T}{2}\right] .
$$

In the time domain, the expression of $x(t)$ is shown in formula (34). $\varphi(t)$ is a time-dependent phase function. Discretize the above formula: the sampling frequency is $F_{s}$, the sampling interval is $T_{s}$, and the number of sampling points is $M=T / T_{s}$, where $T$ is the total duration time of the waveform transmission process. Then, we can get $x(n)$ : 


$$
x(n)=c\left[e^{j \varphi_{0}}, e^{j \varphi_{1}}, e^{j \varphi_{2}}, \ldots, e^{j \varphi_{M-1}}\right]^{T} .
$$

The phase of $x(n)$ can be written as $\varphi=$ $\left[\varphi_{0}, \varphi_{1}, \varphi_{2}, \ldots, \varphi_{M-1}\right]^{T}$. By phase modulation, it is assumed that the energy spectral density of the transmit waveform at this time is $\varepsilon_{\mathrm{pm}}(f)$. After discrete sampling, the energy spectral density becomes $\varepsilon_{\mathrm{pm}}(k)$. After $x(n)$ performing $N(M \ll N)$ point discrete Fourier transform, the energy spectral density can be expressed as

$$
\varepsilon_{\mathrm{pm}}(k)=|X(k)|^{2} .
$$

We suppose that the number of samples is $N$, and the energy spectrum density function of the transmit waveform to be synthesized is $|X(k)|^{2}, k=0,1,2, \ldots, N-1$, where $|X(k)|^{2}$ is the discrete synthetic waveform energy spectral density. Considering generality, we assume that the constant envelope constraint of the waveform is 1 . Using discrete complex signals $x(n)$ with length $M$ to represent timedomain signals, where $n=0,1,2, \ldots, M-1$, the $N$-point Discrete Fourier Transform (DFT) of $x(n)$ is

$$
X(k)=\sum_{n=0}^{M-1} x(n) e^{-j 2 \pi k n / N} .
$$

At this time, the constant envelope time-domain form of the optimal waveform is $x(n)$. This will involve the Fourier transform in the time and frequency domain, which is

$$
x(n)=F^{-1}[X(k)] .
$$

Normalize the energy of the time-domain signal $\overline{x(n)}=x(n) /|x(n)|$, where $F^{-1}$ represents the inverse Fourier transform. The energy spectral density of the timedomain signal obtained by this method is quite different from the required energy spectral density. A commonly used technology is the phase stationary method, which uses the principle of phase stationary; that is, most of the energy of the signal is concentrated near the stationary phase point. An iterative method is used to synthesize the time-domain signal $x(t)$.

According to the method mentioned in the multitarget water-filling algorithm previously in Section 3, we can know that the optimal energy spectral density of the transmit waveform is $\varepsilon_{\text {opt }}(f)$. Discretizing the optimal energy spectral density, we can obtain $\varepsilon_{\text {opt }}(k)$.

Based on the water-filling algorithm, we propose the idea of adding a phase. After adding the phase, it can make the energy spectrum of the transmit waveform as close as possible to the optimal transmit waveform spectrum. In other words, it can make the mean square error of the two waveforms as small as possible. The optimal phase $\varphi_{k}$ is obtained through the idea of loop iteration, and the timedomain expression of the transmit waveform $x(n)$ is finally determined. Under the above assumptions, the radar transmit waveform based on phase modulation is obtained. The core idea is time-domain synthesis based on information theory. Next step, we will discuss the three cases separately. The flowchart is shown in Figure 3.

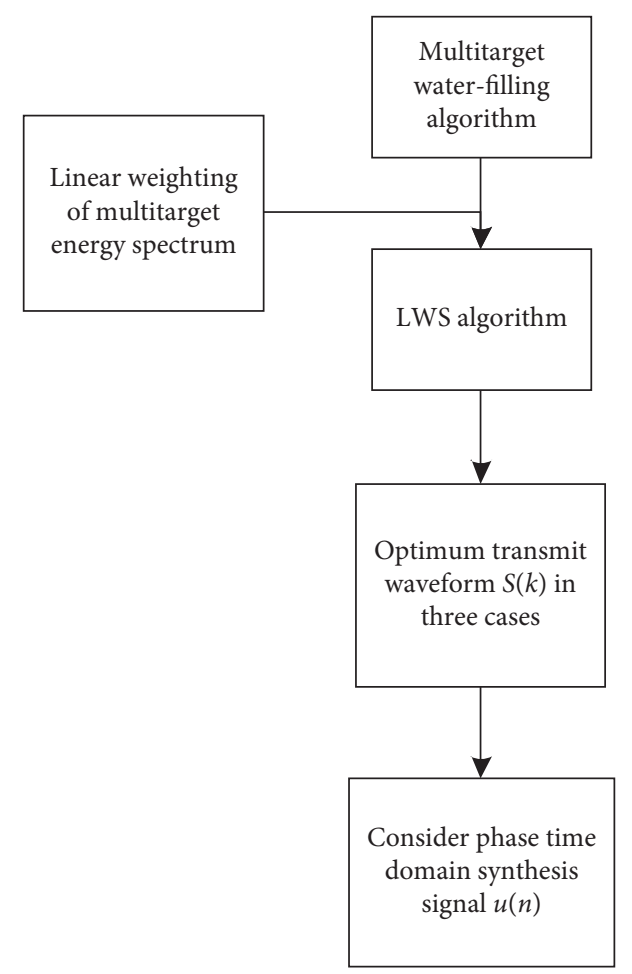

FIgURE 3: Multitarget transmit waveform algorithm.

Based on the multitarget radar transmit waveform, the paper combines the idea of cyclic iterative solution and minimum mean square error to find the optimal synthetic waveform $x(n)$. Let the amplitude spectrum of $X(k)$ and the optimal transmit signal $D(k)$ as close as possible [6], and assume that $\varepsilon_{\text {opt }}(k)=|X(k)|^{2}$, where

$$
x(n)=F^{-1}\left(\sqrt{\varepsilon_{\mathrm{opt}}(k)}\right) .
$$

We assume $\varepsilon_{\text {opt }}(k)=D(k), k=0,1,2, \ldots, N$, where $D(k)=[D(1), D(2), D(3), \ldots, D(N-1)]^{T}$. It can be known from the above analysis that it is necessary to find a suitable cost function. Combined with the analysis of the previous content, we can know that the minimum mean square error-based cost function can be expressed as

$$
H(\varphi)=\sum_{k=0}^{N-1}[D(k)-|X(k)|]^{2},
$$

where $X(k)$ is the discrete Fourier transform of $x(n)$. Assume that the phase of $X(k)$ is $\theta(k)$, and the expression is $X(k)=|X(k)| e^{j \theta(k)}$. Thus, the above formula (40) can be expressed as

$$
H(\varphi)=\sum_{k=0}^{N-1}\left|D(k) e^{j \theta(k)}-X(k)\right|^{2} .
$$

We suppose $q=\left[D(0) e^{j \theta_{0}}, D(1) e^{j \theta_{1}}, D(2) e^{j \theta_{2}}, \ldots\right.$, $\left.D(N-1) e^{j \theta_{N-1}}\right]^{T}$, and $W$ is a Discrete Fourier Transform (DFT) matrix with $N \times M$. Using the principle of discrete Fourier transform, we can obtain 


$$
X(k)=\sum_{n=0}^{N-1} x(n) W_{N}^{n k}, \quad k=0,1,2, \ldots, N-1,
$$

where $\left(W_{N}\right)^{n k}=\left(e^{-j 2 \pi / N}\right)^{n k}$. With the Fourier transform principle, the transformation matrix $W$ used in this paper is set to

$$
W=\left[\begin{array}{cccc}
w^{0,0} & w^{0,1} & \ldots & w^{0, M-1} \\
w^{1,0} & w^{1,1} & \ldots & w^{1, M-1} \\
& \ldots & \ldots & \\
w^{N-1,0} & w^{N-1,1} & \ldots & w^{N-1, M-1}
\end{array}\right]
$$

Therefore, from the above analysis, we can know that after double samplings, the row and column transformation is performed by $W$, and finally we get

$$
X=W x .
$$

After this conversion, the cost function, which is the minimum mean square error, can be expressed as

$$
H(\varphi)=(q-W x)^{H}(q-W x),
$$

where $H$ represents conjugate transpose. This is a nonlinear least square error estimation problem. Using the linear minimum estimator in the mathematical estimation method, we can finally find the minimum estimator of the time-domain waveform $x$ :

$$
\widehat{x}=\frac{1}{N} W^{H} q .
$$

The phase $\theta(k)=[\theta(0), \theta(1), \theta(2), \ldots, \theta(N-1)]$ of the vector $q$ is determined by the discrete Fourier transform $X(k)$ of the transmit signal $x(n)$. The phase $\theta_{k}$ is solved using a cyclic iteration method. Because the minimum estimator is processed iteratively as given in formula (46), it cannot be guaranteed that the amplitude of $\hat{x}$ be constant during the whole transmission process. It needs to be normalized during iteration, so $\overline{x(n)}=x(n) /|x(n)|$.

Figure 4 is the flowchart of constant-envelope timedomain synthetic signal algorithm based on multitarget radar transmit waveform.

The phase iteration process of the time-domain composite signal based on phase modulation is as follows:

(1) Initialize the number of iterations $l, \theta^{l}, q^{l}=$ $\left[D(0) e^{j \theta_{0}}, D(1) e^{j \theta_{1}}, D(2) e^{j \theta_{2}}, \ldots, D(N-1) e^{j \theta_{N-1}}\right]^{T}$.

(2) Calculate $\widehat{x}^{l+1}=1 / N W^{H} q^{l}$.

(3) In order to satisfy that the amplitude of the transmit signal is 1 , perform normalization processing and calculate $\overline{\hat{x}}^{l+1}=\widehat{x}^{l+1} /\left|\widehat{x}^{l+1}\right|$.

(4) Calculate $\bar{X}^{l+1}=W \overline{\hat{x}}^{l+1}$.

(5) Calculate $\bar{X}^{l+1}$ of phase $\theta^{l+1}$ and error $\Delta=\sum_{l=0}^{M-1}\left|\overline{\widehat{x}}^{l+1}-\overline{\widehat{x}}^{l}\right|, \Delta \leq \gamma$. If $H(\varphi) \leq \Delta$, the loop is ended. It is the time-domain signal $\overline{\hat{x}}^{l+1}$ for which the solution is required; otherwise, the next loop is entered, where $\overline{\widehat{x}}^{l}$ is the $l$ estimate of $x(n)$.

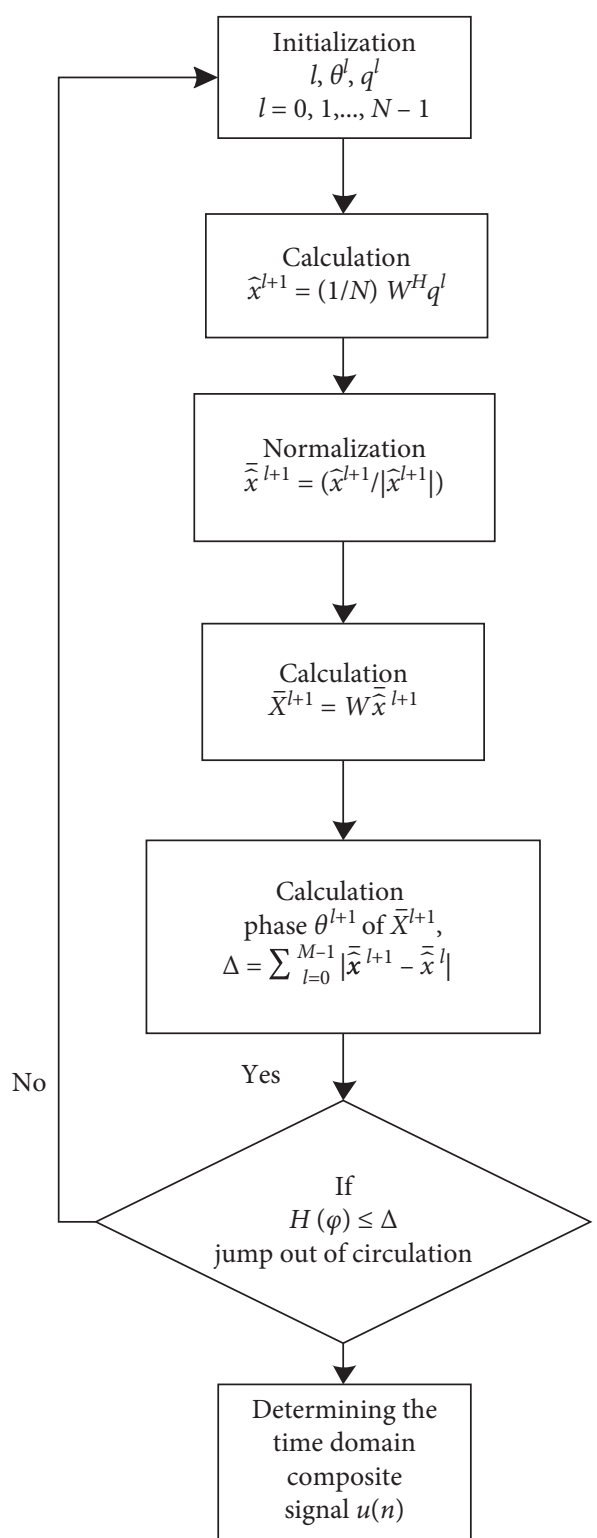

FIgURE 4: Flowchart of time-domain synthesis algorithm.

4.2. Computational Complexity Analysis. First of all, the multitarget radar waveform design process based on information theory mainly involves calculation based on mutual information and signal to interference plus noise ratio. Because the waveform function involved in this paper is a concave function, the Lagrangian multiplier method is used to derive accordingly the specific derivation process explained in detail. As the expression of the radar transmit waveform is finally obtained by two partial derivatives, the analysis shows that the time complexity here is $O(n)$.

Secondly, for the time-domain synthetic waveform, adding phase information at this time, we design the timedomain synthetic waveform with the aid of the radar's optimal transmit waveform. This process uses the knowledge points in the communication principle. 
Using discrete complex signal $x(n)$ with length $M$ to represent time-domain signals, where $n=0,1,2, \ldots, M-1$, the $N$-point Discrete Fourier Transform (DFT) of $x(n)$ is

$$
X(k)=\sum_{n=0}^{M-1} x(n) e^{-j 2 \pi k n / N} .
$$

At this time, it appears the constant envelope timedomain form of the optimal waveform. This will involve the Fourier transform in the time and frequency domain, which is

$$
x(n)=F^{-1}[X(k)]
$$

Adding a conversion matrix, the minimum mean square error (MMSE) is used to design the waveform

$$
\begin{aligned}
W & =\left[\begin{array}{cccc}
w^{0,0} & w^{0,1} & \ldots & w^{0, M-1} \\
w^{1,0} & w^{1,1} & \ldots & w^{1, M-1} \\
& & \ldots & \\
w^{N-1,0} & w^{N-1,1} & \ldots & w^{N-1, M-1}
\end{array}\right], \\
H(\varphi) & =(q-W x)^{H}(q-W x) .
\end{aligned}
$$

Finally, the phase information of the waveform is determined through a cyclic iteration process to achieve the purpose of obtaining more target information. Here, the loop is used to superimpose the calculation, and the phase information is obtained through MMSE. Through code analysis, the time complexity involved here is $O(n)$.

\section{Simulation Results and Analysis}

Based on the theoretical derivation and analysis in Sections 3 and 4, the feasibility and practicability of the algorithm are further verified by simulations. Parameter settings are presented in Table 1.

Assume system bandwidth $B=10 \mathrm{MHZ}$, and system transmit energy $E=10^{3} \mathrm{~J}$. The frequency $f$ takes the value within $[0, B]$. Figure 5 shows the distribution of clutter energy spectral density within the normalized frequency, where $S_{c c}(f)=\mid 0.2(\cos (0.3 \pi(f+0.35)))+0.5(\sin (0.8 \pi$ $(f+0.3)))-0.15 \mid+0.01$. Clutter is a mixed trigonometric function.

Suppose that there are 4 targets, and the weighting coefficients have the relationship $\sum_{l=1}^{L} w_{l}=1$. The four targets are distributed independently, and the known targets have approximately bandpass-shaped spectra. The clutter is cosine-shaped, and noise is AWGN. The target energy spectrum variance (ESV) involved in the treatment process is the weighted target energy spectrum variance.

By considering the three cases in Section 3, the objective function and the optimal transmit waveform energy spectral density can be obtained under the corresponding criteria, that is, $\varepsilon_{\text {opt }}(f)$. After obtaining the optimal energy spectral density of the transmit signal, sampling is performed. The number of sampling points $N$ is 5000, and the length $M$ of the time-domain signal to be synthesized $x(n)$ is 500 . The allowable error is set to $\gamma=0.01$.

\subsection{Radar Waveform Design for Stochastic Multitarget Based on Information Theory}

5.1.1. Design of Radar Waveform Based on Mutual Information in Noise Only. We first consider the design of the radar transmit waveform based on mutual information in the case of noise only. Figure 5 shows the target spectrum of four known targets in the research process, which obeys the similar distribution within the normalized frequency. The time-domain synthetic method proposed in this paper is used to further perform $x(n)$ in time-domain synthesis. Figure 6 is the total target spectrum within the normalized frequency after multitarget weighting during the experiment. Figure 7 is a comparison of the total target spectrum and the optimal transmit waveform spectrum. It can be seen that, based on the trend consistency, the amplitude of the waveform spectrum changes due to the influence of the transmit energy.

The length $M$ of the time-domain signal $x(n)$ to be synthesized is 500 . Figure 8 shows the phase information of the time-domain synthetic signal $x(n)$, including real part, imaginary part, phase, and amplitude distribution. After adding the phase information, the signal is determined completely. It can be seen from Figure 8 that the amplitude value of the composite signal is constant 1 , which verifies the characteristics of the constant amplitude of the composite signal during the research process.

Figure 9 shows the comparison of time-domain synthetic waveform spectrum and optimal transmit waveform spectrum. Figure 9 shows that when only noise is considered, the energy spectrum is evenly distributed in the entire frequency spectrum, instead of being concentrated in a part. This allows energy to be reasonably distributed and improves energy utilization during transmission process. Through the comprehensive analysis of Figures 7 and 9, it can be seen that compared to the energy spectral density of the optimal transmit signal in the time-domain synthetic signal, the curve trends of these two signals are basically the same, indicating that the idea of using the mean square error can make the two achieve high fitting.

With the method of time-domain waveform synthesis in Section 4, the allowable error $\gamma$ is 0.01 . Figure 10 shows that when the cut-off allowable error is set, only the phase information when iterating for 18 times in the case of noise is set. Then, calculate phase of $x(n)$, and the error is 0.0087474 . This algorithm uses the data to explain the problem and uses the parameter $H(\varphi)$ to evaluate. As shown in Figure 10, it can be seen that as the number of iterations increases, the error will become smaller and smaller. It eventually approaches 0.01 and finally stops iteration. The time-domain synthetic signal is the closest to the optimal transmit waveform, which further proves the significance of the study.

5.1.2. Design of Radar Waveform Based on Mutual Information in the Presence of Clutter. On the basis of only considering the existence of noise, we further study radar transmit waveform design based on mutual information in the presence of clutter. The clutter and weighted multitarget 
TABLe 1: Parameter settings.

System bandwidth

System transmit energy

Frequency

Clutter energy spectral density

Number of targets

Weighting coefficients

Sampling points

Length of the time-domain signal

Allowable error

$B=10 \mathrm{MHZ}$
$E=10^{3} \mathrm{~J}$
$[0, B]$
$S_{c c}(f)=|0.2(\cos (0.3 \pi(f+0.35)))+0.5(\sin (0.8 \pi(f+0.3)))-0.15|+0.01$
4
$\sum_{l=1}^{L} w_{l}=1$
5000
500
$\gamma=0.01$

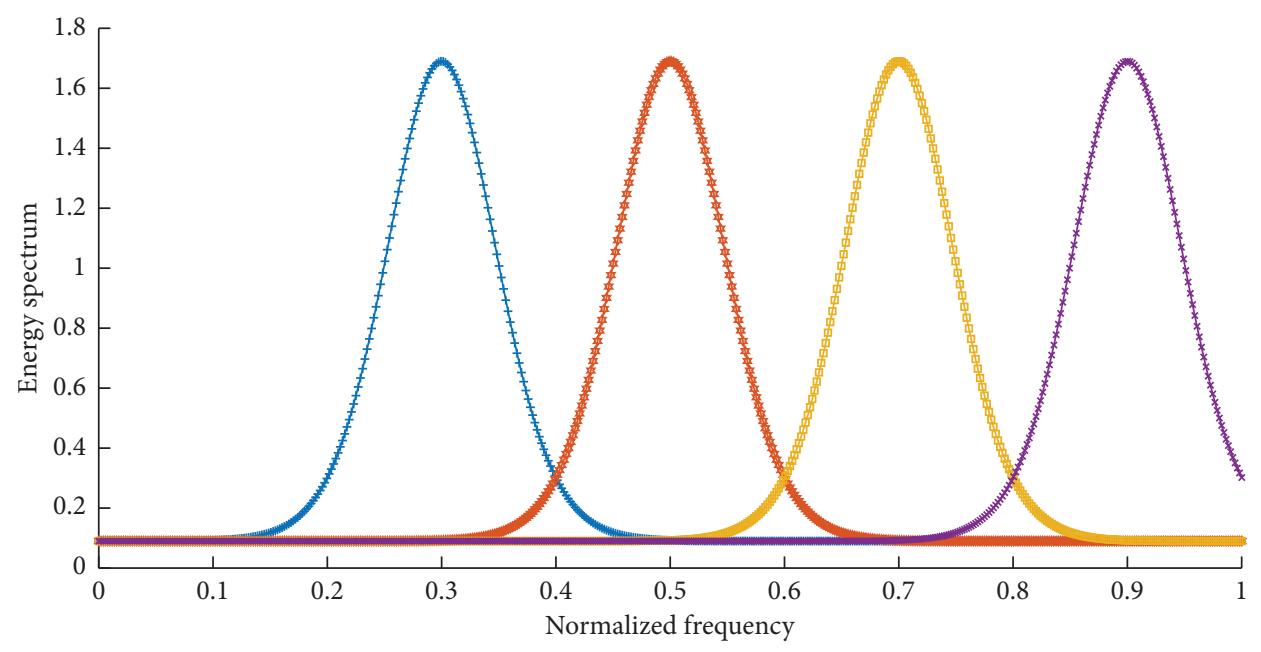

$\begin{array}{ll}- \text { The known target one } & - \text { The known target three } \\ - \text { The known target two } & -* \text { The known target four }\end{array}$

FIgURE 5: Multitarget spectrum.

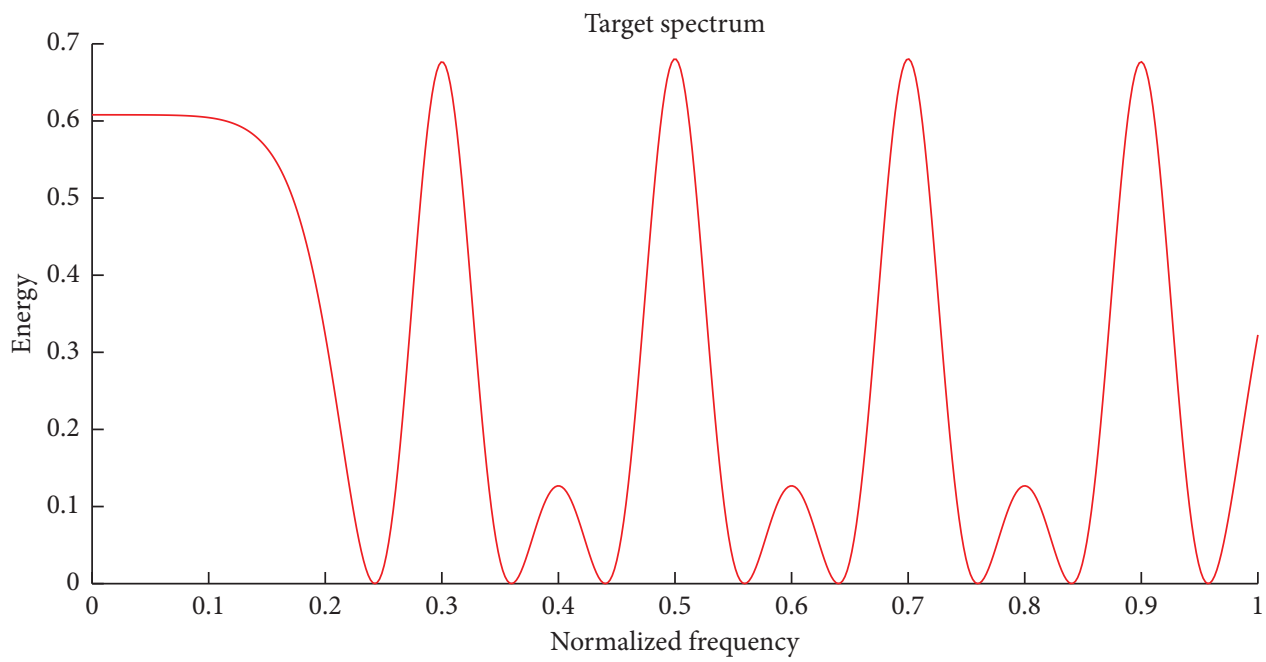

— Target spectrum

FIgURE 6: Weighted multitarget total target spectrum.

energy spectral density are shown in Figure 11. It can be seen that the setting of the multitarget spectrum is the same as that in the presence of noise only. The clutter spectrum is relatively high at both ends, while it is low at frequency between 0.7 and 0.8 .
In the presence of clutter, the research environment is relatively complicated. At this time, the optimal transmit waveform will be interfered by noise and clutter at the same time. According to the theoretical research of radar transmit waveforms, it is known that the optimal transmit waveform 


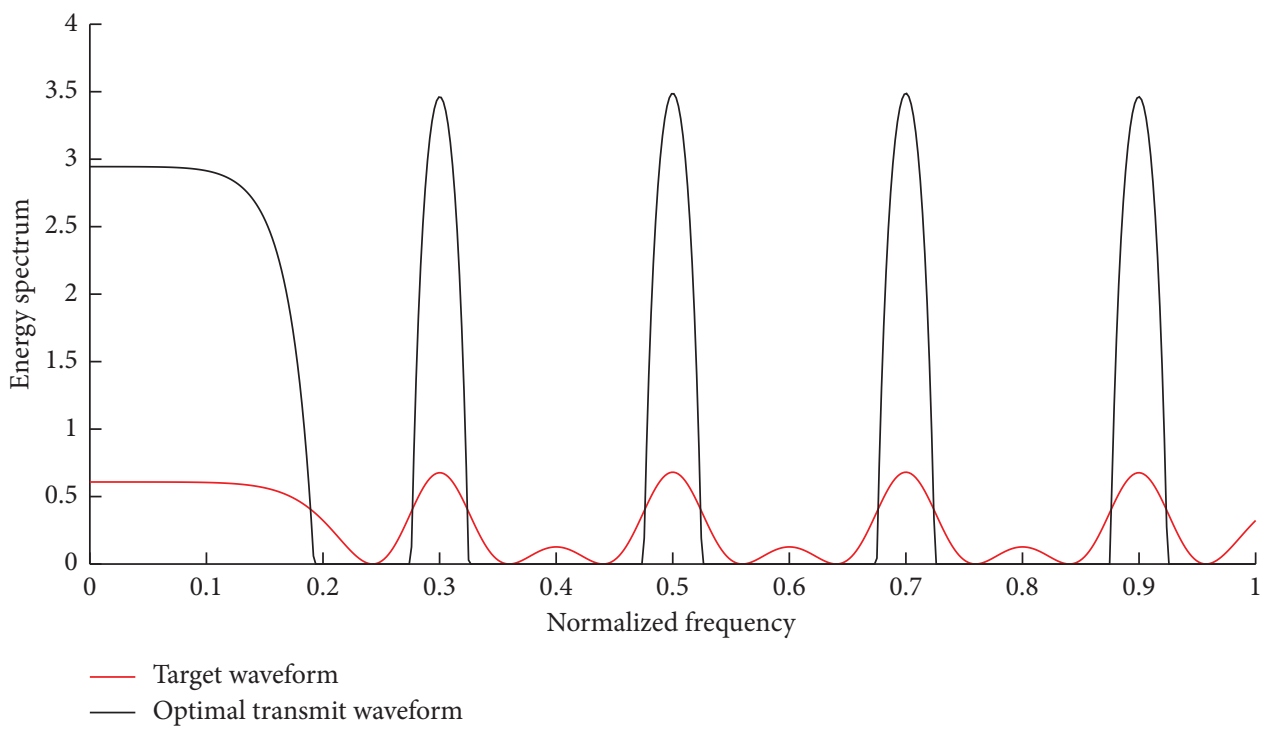

FIGURE 7: Target spectrum and optimal transmit waveform spectrum based on mutual information in only noise.
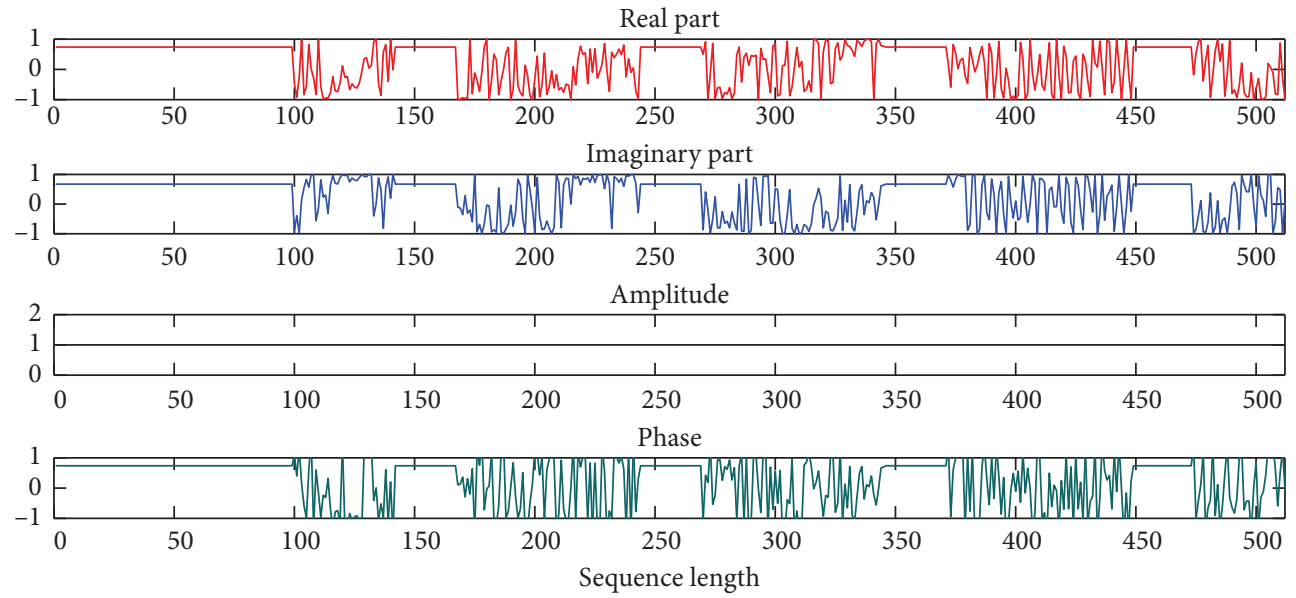

Figure 8: Time-domain synthesis signal $x(n)$ based on mutual information.

is designed under the energy constraints. Meanwhile, the principle of clutter compensation is used. Figure 12 shows that, in order to maximize the mutual information between the target and the echo, it is reasonable to allocate energy; that is, more energy is distributed in places with less interference clutter.

Figure 13 shows the relevant parameters of the synthetic signal $x(n)$ after the loop iteration, including the phase, amplitude, and distribution of the real and imaginary parts with a sequence length of 500 . The length $M$ of the timedomain signal $x(n)$ to be synthesized is 500 . It can be seen that the characteristics of the constant amplitude of the signal $x(n)$ are guaranteed during the treatment process, and the phase information and the real part information are basically the same. The imaginary part is basically not considered during the setting process impact.

In the case of multiple targets, the idea of adding phase is obtained by finding the minimum mean square error of the energy spectrum $H(\varphi)$ of the time-domain synthetic signal $x(n)$ and the optimal transmit waveform $\varepsilon_{\text {opt }}(f)$. The smaller the mean square error is, the closer the two waveforms are, which can explain the effectiveness of the research after adding the phase. Figure 14 shows the energy spectrum density distribution of the time-domain synthetic signal $x(n)$ and the optimal transmit waveform $\varepsilon_{\text {opt }}(f)$. It can be seen that due to the influence of clutter, the energy spectrum is not distributed at all frequencies, while it is distributed in the place where there is less clutter distribution and the target spectrum is relatively high. Using the principle of clutter compensation will make the information not being lost as much as possible during the transmission. The mutual information between the target and the echo is improved during transmission. The optimal time-domain synthetic signal $x(n)$ is basically consistent with the optimal transmit waveform $\varepsilon_{\text {opt }}(f)$ in trend after the loop iteration.

The feasibility of the algorithm is verified through the previous simulations. By performing $x(n)$ in time-domain synthesis, the energy spectral density of the time-domain 


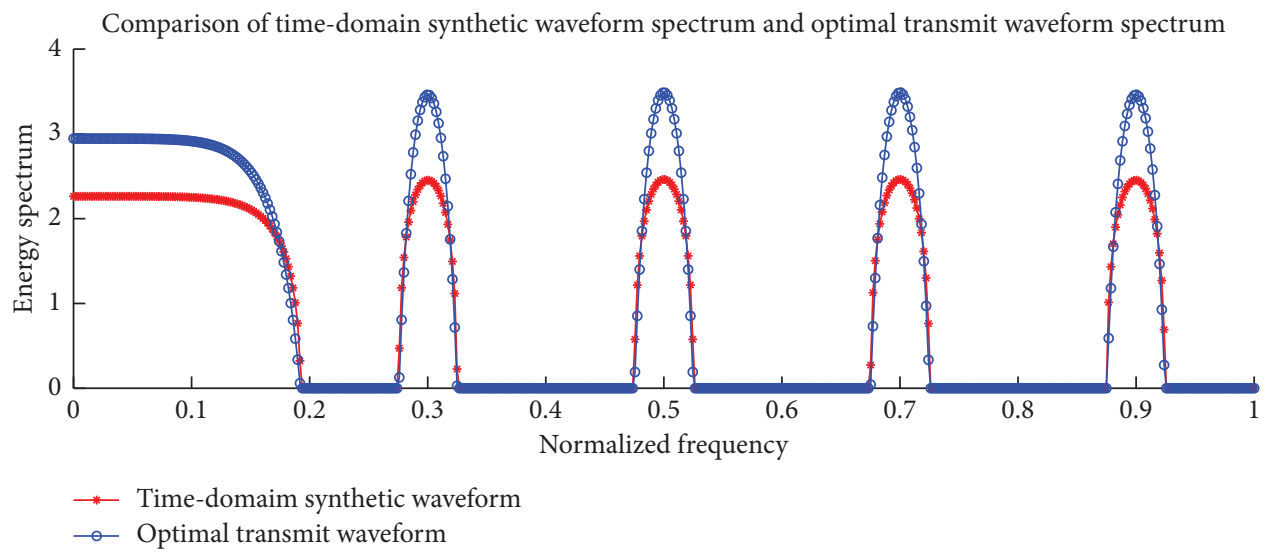

Figure 9: Time-domain synthetic waveform spectrum and optimal transmit waveform spectrum.

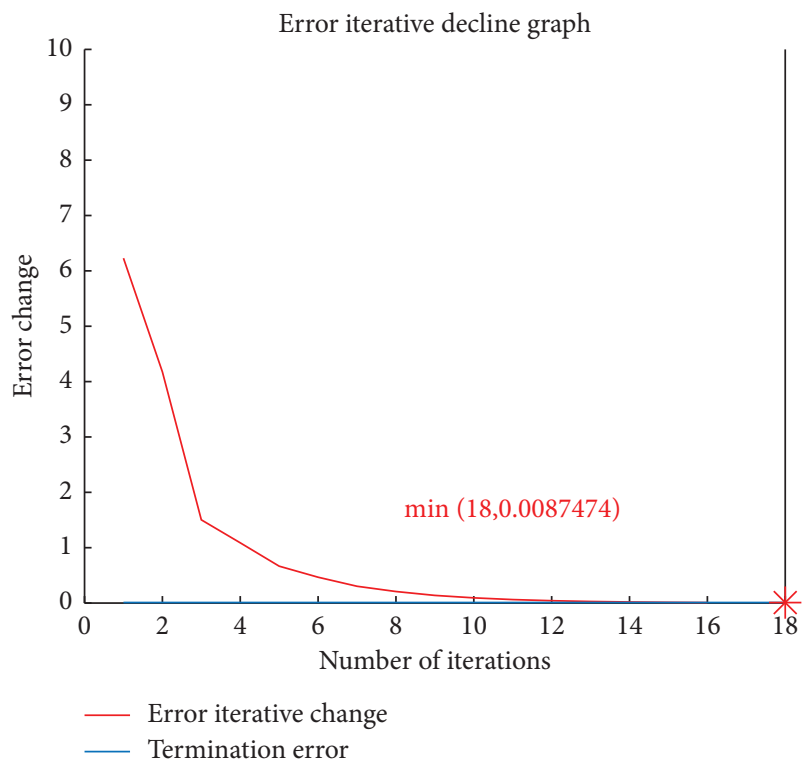

FIGURE 10: Relationship between error variation and the number of iterations.

synthetic signal is closer to the energy spectrum of the optimal transmit waveform, which is evaluated with parameters $H(\varphi)$. Figure 15 shows that as the number of iterations increases, in the case of $\gamma=0.01$, error will become smaller and smaller and eventually approaches 0.01 . Finally, it jumps out of the loop after the 18th iteration, with an error value of 0.008407 , and stops the iteration. At this time, the optimal transmit waveform is the closest to the time-domain synthetic signal.

5.1.3. Design of Radar Waveform Based on Signal-to-Interference Ratio in the Presence of Clutter. The above two sections consider the comparison of the LWS algorithm with the presence or absence of clutter under the mutual information criterion and the time-domain synthetic algorithm after adding the phase information. This section analyzes the time-domain synthetic algorithm based on the signal-tointerference ratio under energy constraints.
Different from the criterion based on mutual information, in the case where waveforms are both affected by clutter, the optimal transmit waveform spectrum based on the signal-to-interference ratio is evenly distributed in all frequencies. However, the optimal transmit waveform spectrum based on the mutual information criterion is distributed where the peak of the target spectrum is high, and energy is basically not allocated in other low places. This is also the reason why the reliability of the system performance can be evaluated based on the signal-to-interference ratio rather than mutual information, so that more target information can be obtained. For multitarget, it is more conducive to target recognition.

Due to the influence of clutter and noise, the total target waveform and the optimal transmit waveform spectrum based on the signal-to-interference ratio for multiple targets are shown in Figure 16. Obviously, the clutter distribution is low and vice versa.

The length $M$ of the time-domain signal $x(n)$ to be synthesized is 500 . Figure 17 is the phase information of the time-domain synthetic signal $x(n)$ based on the signal-tointerference ratio, including the real part, imaginary part, phase, and amplitude distribution. It can be seen from Figure 17 that the amplitude value of the composite signal $x(n)$ is constant 1 , which verifies the characteristics of the constant amplitude of the composite signal.

In the case of multitarget, the idea of adding phase is obtained by finding the minimum mean square error $H(\varphi)$ between the energy of the time-domain synthetic signal $x(n)$ and the optimal transmit waveform $\varepsilon_{\text {opt }}(f)$. The smaller the mean square error $H(\varphi)$ is, the closer the two waveforms are, which can explain the effectiveness of the research after adding the phase information. Figure 18 shows the energy spectrum density distribution of the time-domain synthetic signal $x(n)$ and the optimal transmit waveform $\varepsilon_{\text {opt }}(f)$. The principle of clutter compensation is used to ensure that information is not lost as much as possible during transmission and to improve the signal-to-interference ratio between the target and the echo during transmission. The optimal time-domain synthetic signal $x(n)$ after the loop iteration is basically consistent with the optimal transmit waveform $\varepsilon_{\text {opt }}(f)$ trend. 


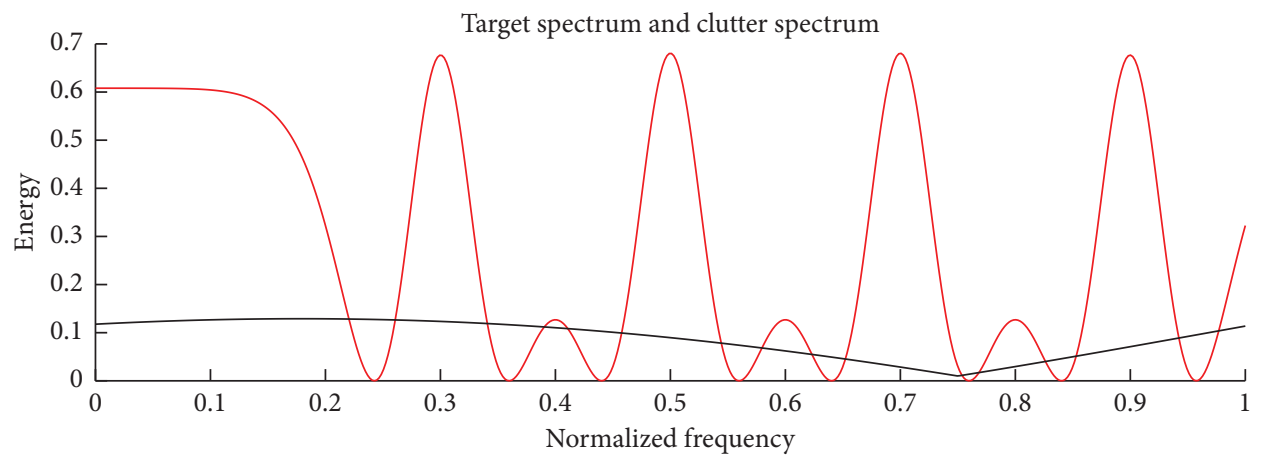

Target spectrum

— Clutter spectrum

FIgURE 11: Target spectrum and clutter spectrum based on mutual information.

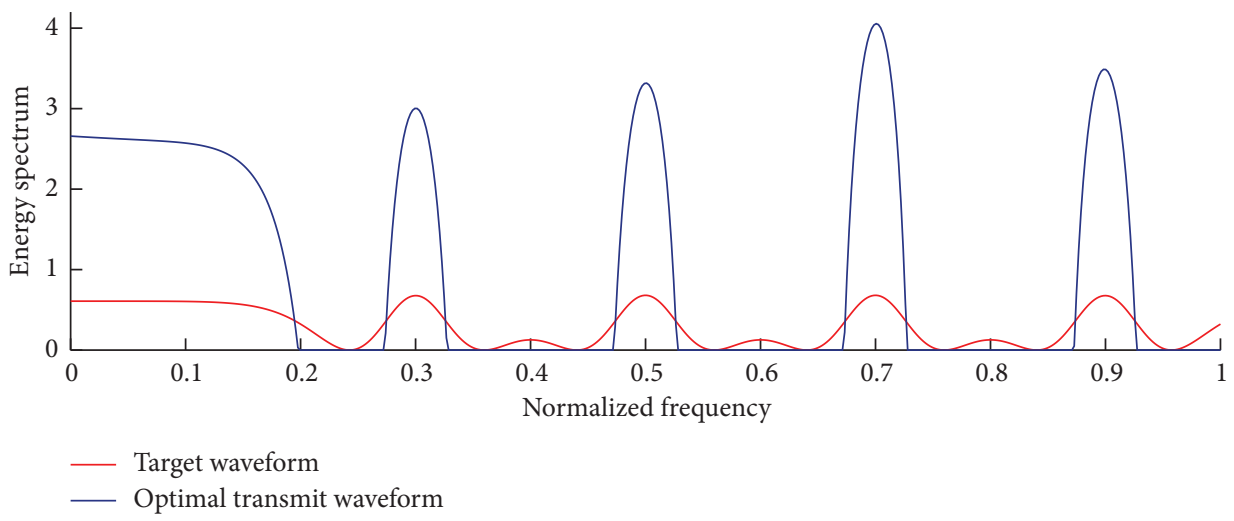

FIGURE 12: Target spectrum and optimal transmit waveform spectrum based on mutual information.
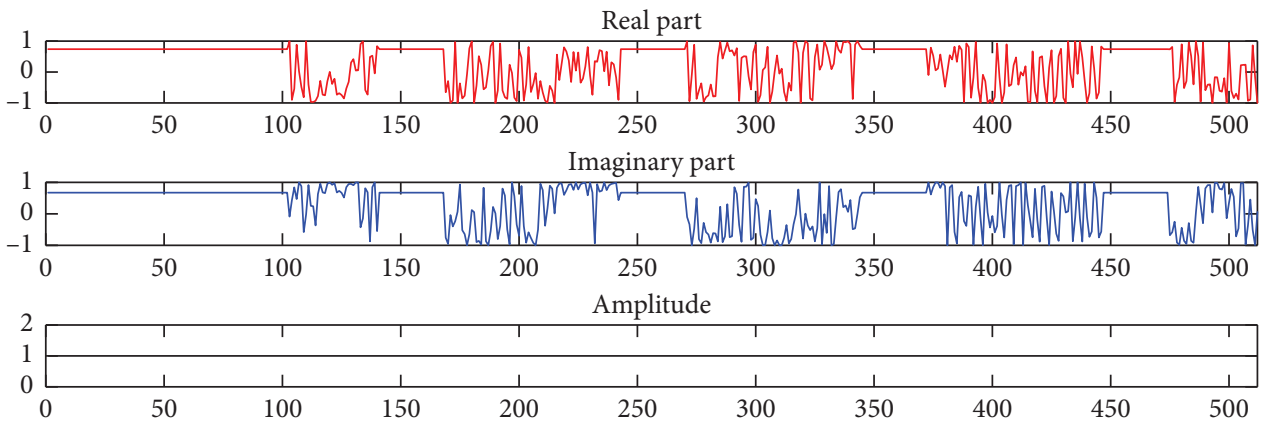

Phase

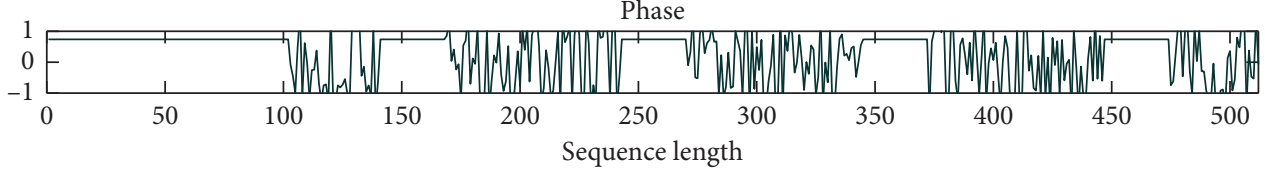

FIGURE 13: Time-domain synthetic signal $\mu(n)$ based on mutual information.

Performing time-domain synthesis by $x(n)$, the energy spectral density of the time-domain synthetic signal $x(n)$ is closer to the energy spectrum of the optimal transmit waveform, which is evaluated with parameter $H(\varphi)$. It can be seen from Figure 19 that as the number of iterations increases, in the case of $\gamma=0.01$, error will become smaller and smaller and eventually approaches 0.01 . At the 17th iteration, the error value is 0.0099628 , and the iteration will finally stop. At this time, the optimal transmit waveform is the closest to the time-domain synthetic signal.

5.2. Comparative Analysis. Through the analysis of the previous sections, the basic idea of the time-domain synthetic algorithm based on mutual information and signal-tointerference ratio is the same. The difference is the 


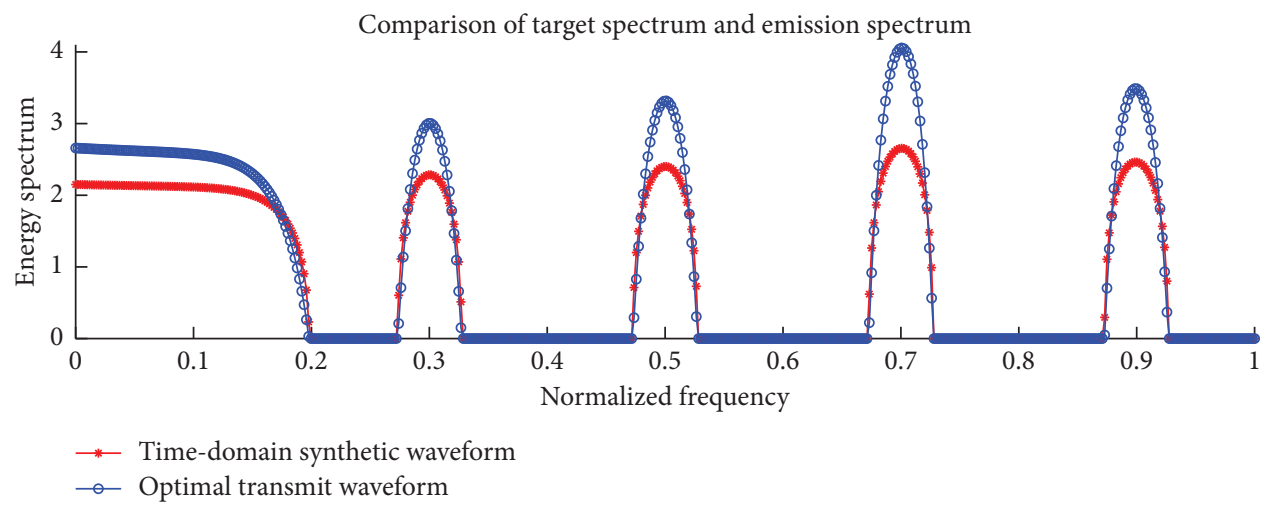

FIGURE 14: Energy spectrum of time-domain synthetic waveform and optimal transmit waveform.

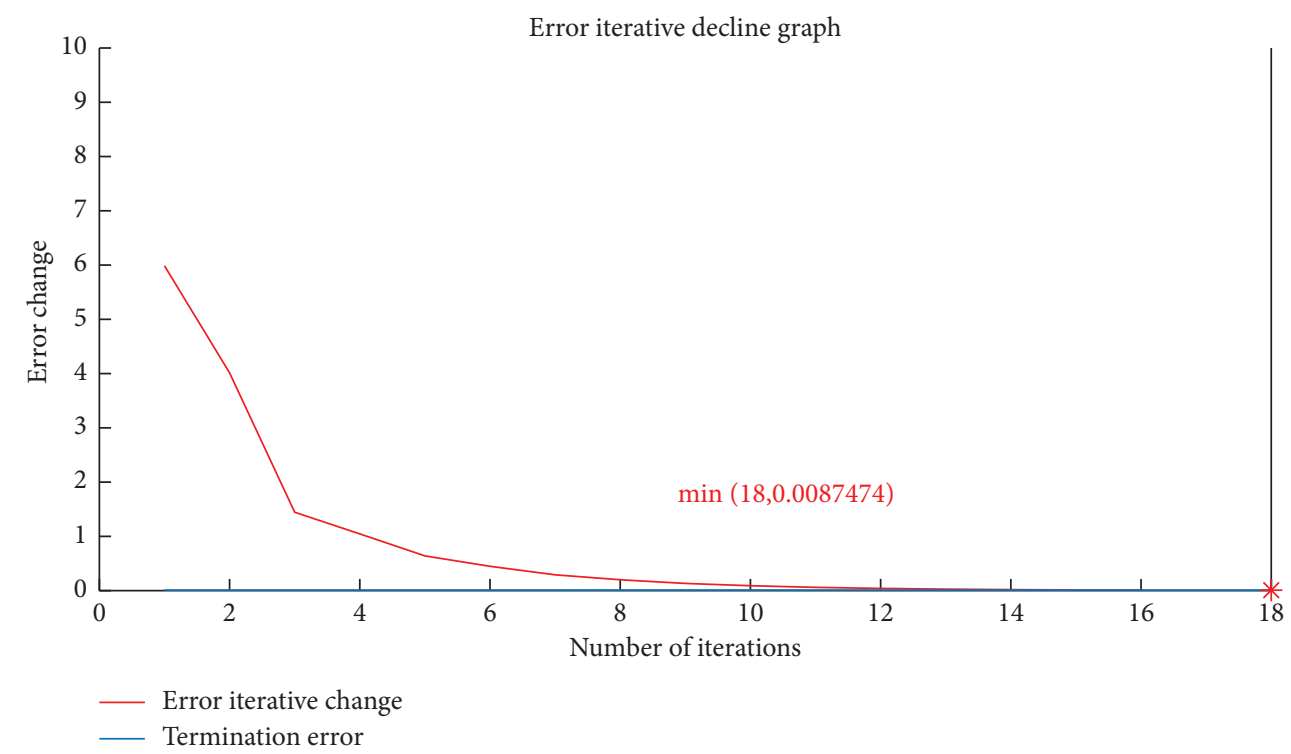

Figure 15: Relationship between error variation and the number of iterations.

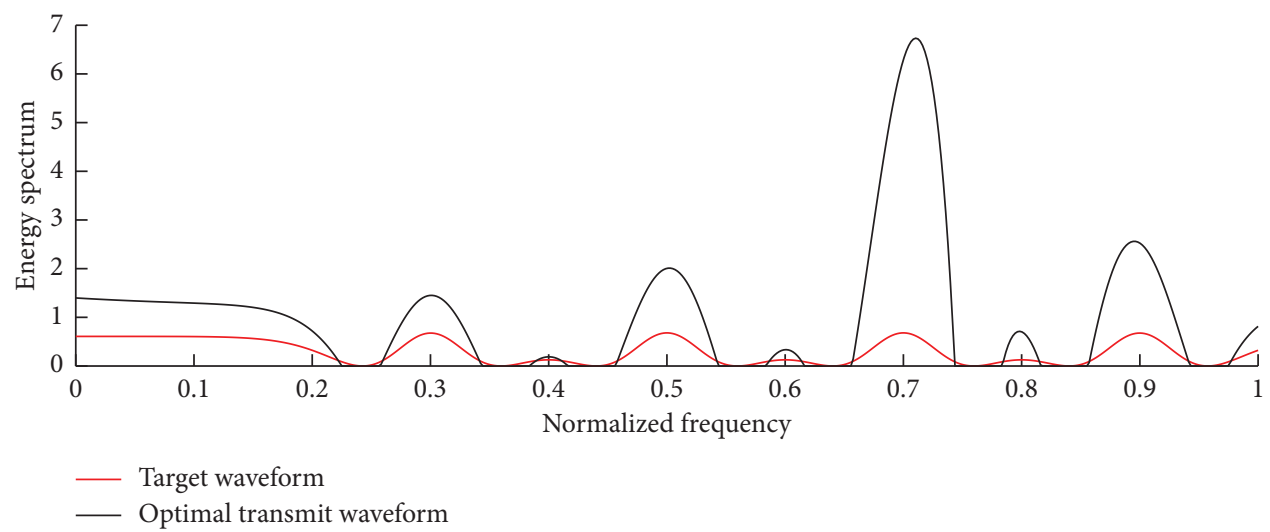

FIGURE 16: Target spectrum and optimal transmit waveform spectrum based on signal-to-interference ratio in the presence of clutter.

expression of the optimal transmit waveform $\varepsilon_{\text {opt }}(f)$ obtained under the LWS algorithm. So the obtained energy spectrum of the time-domain synthetic waveform is completely consistent with the trend of the energy spectrum of the optimal transmit waveform, although the spectrum height is different. This is also due to the relationship between the optimal transmit waveform spectrum and the time-domain synthetic waveform spectrum during the treatment process as shown in formula (39). 

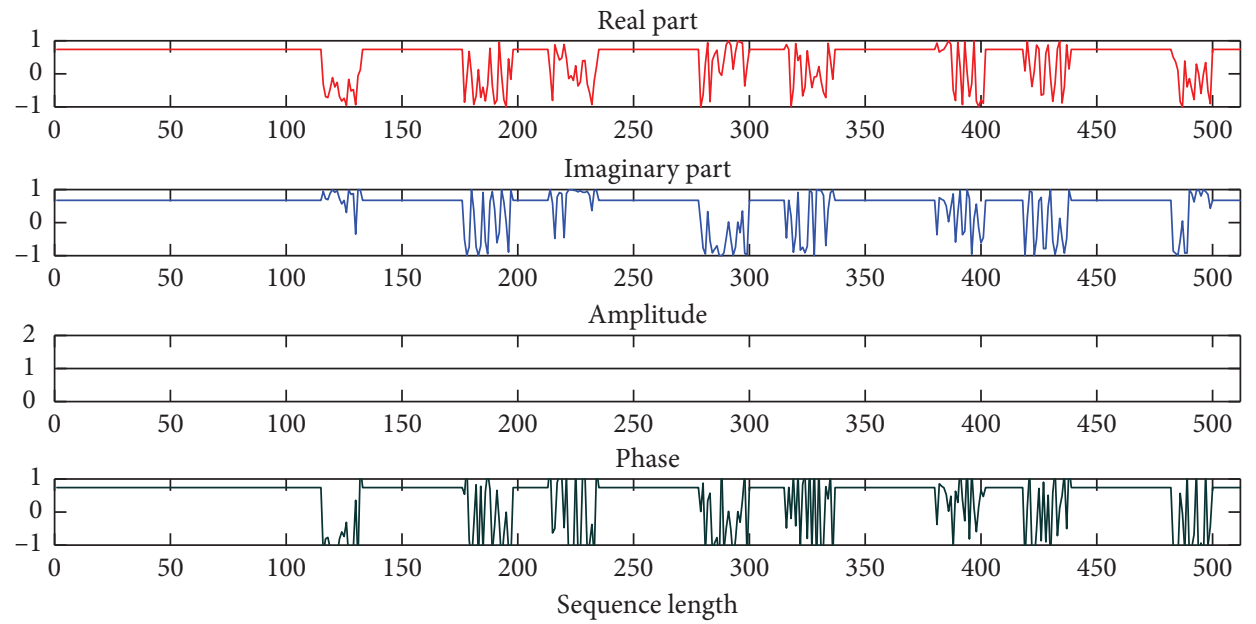

FIgURE 17: Time-domain synthetic signal based on signal-to-interference ratio.

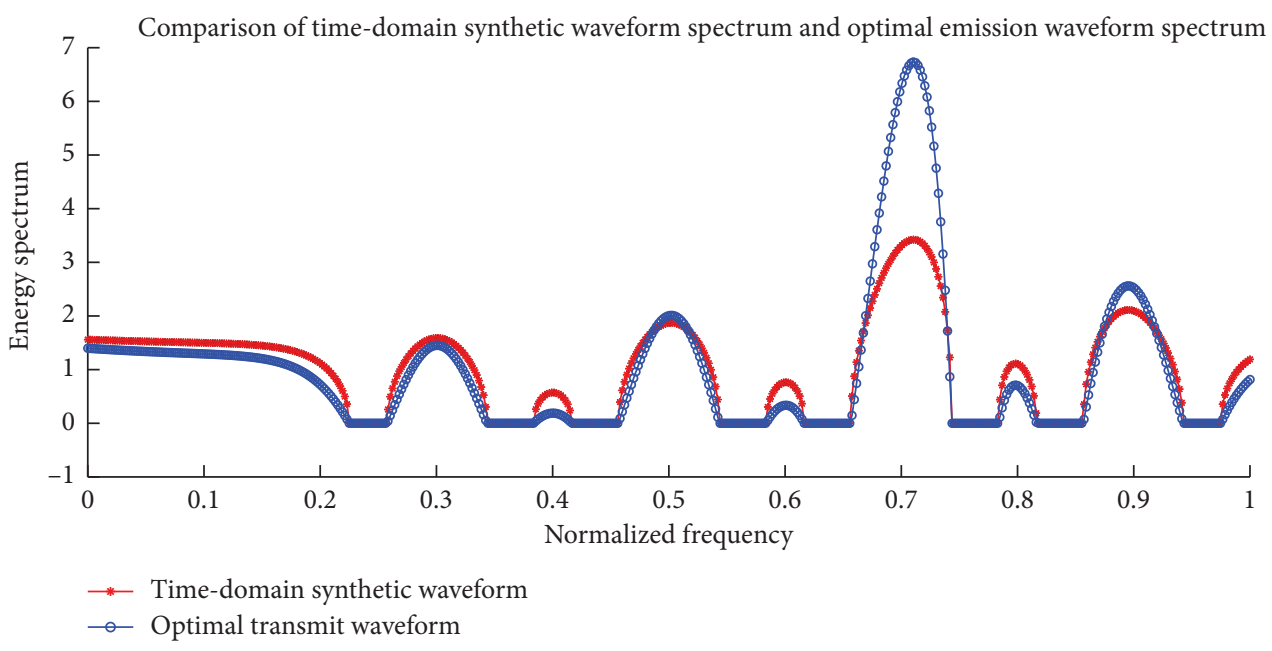

FIGURE 18: Energy spectrum of time-domain synthetic waveform and optimal transmit waveform.

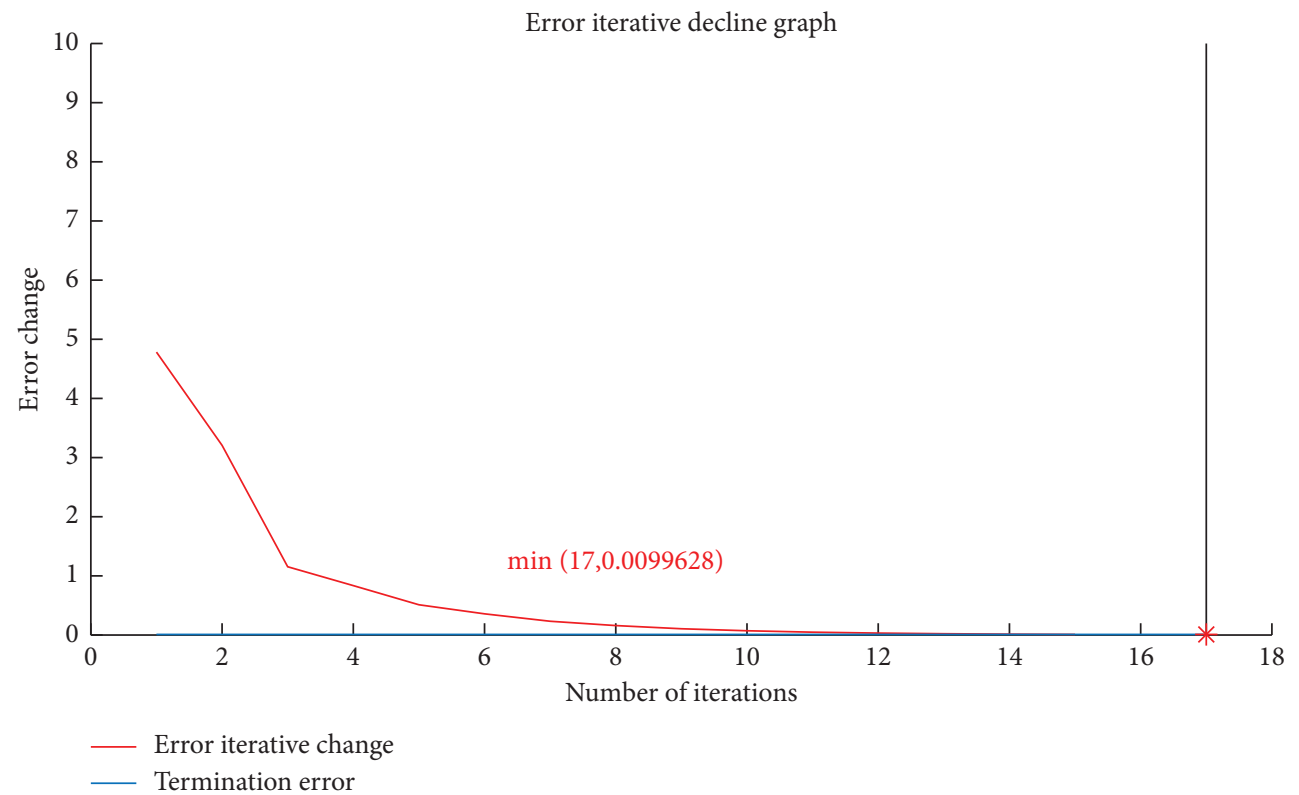

FIGURE 19: Relationship between error variation and the number of iterations. 


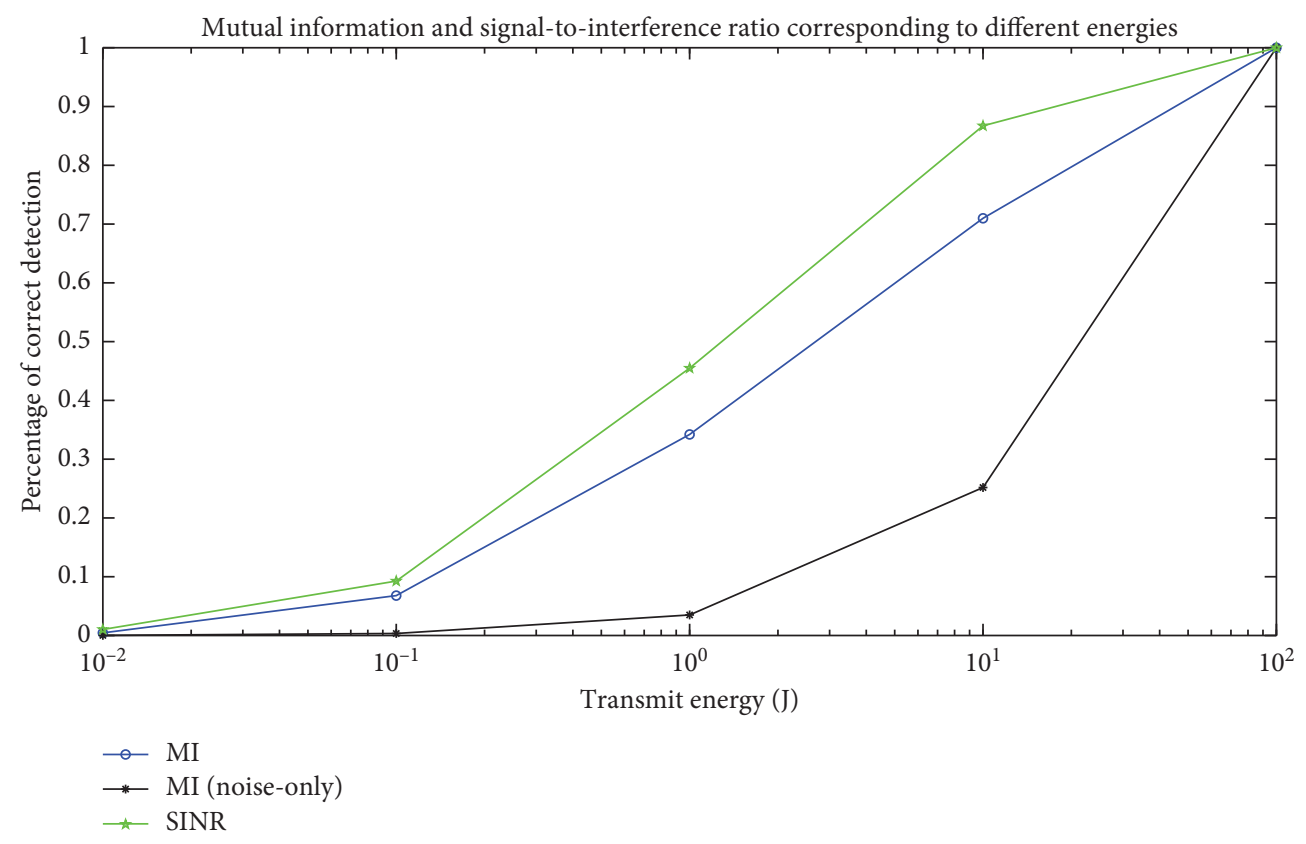

Figure 20: Comparison of detection probability based on three cases.

Figure 20 shows the comparison of detection probability based on three cases. One case is based on the signal-tointerference ratio and the other two cases are based on mutual information whether considering the existence of clutter. In the comparison of detection probability, we can know that the detection probability performance based on the signal-tointerference ratio is the best, and this is also the main reason why a lot of researches focus on the signal-to-interference ratio. It can be further considered that the size of the signal-tointerference ratio can test the performance of the radar system, which has very important research significance.

\section{Conclusions}

There is still a lot of in-depth research on adaptive waveform design based on information theory and its application in cognitive system. Considering information theory, this paper expands from a single target to multiple targets. Based on the characteristics of transmitting in the same beam, each target is reasonably allocated with appropriate corresponding weights. Because each transmit waveform design is optimal for one target, it is not necessarily optimal for other targets. Therefore, based on this consideration, we analyze all targets' weighted energy spectra as a whole.

Based on the traditional water-filling algorithm, this paper considers three cases of LWS algorithm. The optimal transmit waveform energy spectrum in different cases is obtained. Adding the idea of phase information and synthesizing constant-amplitude time-domain signals, a new method is proposed to study the design of the transmit waveform based on the LWS algorithm. Through multiple discrete Fourier transforms, the expression of the time-domain synthetic signal in the frequency domain is obtained. With the idea of the minimum mean square error (MMSE), the energy spectral density of the designed algorithm is as small as possible. Simulation results show that, after multiple iterations, the designed transmit waveform is very close to the optimal transmit waveform. The algorithm proposed in this paper adds the idea of phase information and can obtain more target information through time-domain synthesis of the transmit waveform. Being able to determine target information more accurately has great research value.

This paper has carried out some in-depth research on the design of radar multitarget transmit waveform based on information theory and solved the problem of multitarget in practice. However, the actual environment is more complicated. The clutter considered in this paper is a Gaussian random distribution clutter, and we can further research other types of clutter. This paper adds phase information, and adding other information will make waveform design closer to actual situation.

\section{Data Availability}

All data used to support the findings of this study are included within the article.

\section{Conflicts of Interest}

The authors declare that there are no conflicts of interest regarding the publication of this paper.

\section{Acknowledgments}

This work was supported by the Natural Science Foundation of Hebei Province (no. F2018501051) and the Fundamental Research Funds for the Central Universities (no. N182304022). 


\section{References}

[1] E. J. Kelly, "An adaptive detection algorithm," IEEE Transactions on Aerospace and Electronic Systems, vol. 22, no. 2, pp. 115-127, 1986.

[2] D. Ciuonzo, D. De Maio, and D. Orlando, "A unifying framework for adaptive radar detection in homogeneous plus structured interference- part II: detectors design," IEEE Transactions on Signal Processing, vol. 64, no. 11, pp. 29072919, 2016.

[3] M. Fan, D. Liao, X. Ding, W. Jiang, and X. Li, "Relationship of target recognition performance and radar waveform parameters," Journal of Electronics, vol. 28, no. 1, pp. 77-86, 2011.

[4] R. A. Romero, J. Bae, and N. A. Goodman, "Theory and application of SNR and mutual information matched illumination waveforms," IEEE Transactions on Aerospace and Electronic Systems, vol. 47, no. 2, pp. 912-927, 2011.

[5] R. A. Romero and N. A. Goodman, "Improved waveform design for target recognition with multiple transmissions," in Proceedings of the 2009 International Waveform Diversity and Design Conference, pp. 26-30, Kissimmee, FL, USA, February 2009.

[6] Y. Wei, H. Meng, Y. Liu, and X. Wang, "Radar phasemodulated waveform design for extended target detection," Tsinghua Science and Technology, vol. 16, no. 4, 2011.

[7] X. Gong, H. Meng, Y. Wei, and X. Wang, "Phase-modulated waveform design for extended target detection in the presence of clutter," Sensors, vol. 11, no. 7, pp. 7162-7177, 2011.

[8] Q. Huang, Y. Li, Y. Zeng, and Y. Fu, "Design and characteristic analysis of multicarrier chaotic phase coded radar pulse train signal," International Journal of Antennas and Propagation, vol. 2014, Article ID 724294, 8 pages, 2014.

[9] X. Gong, H. Tan, H. Meng, and J. Guo, "Optimization of radar phase-coded signals for multiple target detection," Signal Processing, vol. 100, pp. 186-196, 2014.

[10] L.-L. Wang, H.-Q. Wang, Y.-Q. Cheng, and Y.-L. Qin, “A novel SINR and mutual information based radar jamming technique," Journal of Central South University, vol. 20, no. 12, pp. 3471-3480, 2013.

[11] L.-L. Wang, H.-Q. Wang, Y.-Q. Cheng, and Y.-L. Qin, "Joint adaptive waveform and baseline range design for bistatic radar," Journal of Central South University, vol. 21, no. 6, pp. 2262-2272, 2014.

[12] B. Jiu, H. Liu, L. Zhang, Y. Wang, and T. Luo, "Wideband cognitive radar waveform optimization for joint target radar signature estimation and target detection," IEEE Transactions on Aerospace and Electronic Systems, vol. 51, no. 2, pp. 1530-1546, 2015.

[13] Y. Dong, Z. Zhang, Y. Zhao et al., "Design of extended target detection waveform based on SCNR cognitive radar," Journal of Computer Applications, vol. 35, no. 7, pp. 2105-2108, 2015.

[14] L. Cao, J. Zhang, X. Wang et al., "Cognitive radar estimation waveform design based on minimum mean-square error," Journal of Detection \& Control, vol. 35, no. 4, pp. 63-67, 2013.

[15] B. Zhu, Y. Gao, H. Sheng, K. Wang, and X. Liu, "Waveform design based multi-target hypothesis testing under unknown clutter parameters," in Proceedings of the IEEE International Symposium on Geoscience and Remote Sensing, pp. 6617-6620, Beijing, China, July 2016.

[16] C. Peng and L. Wu, "Waveform design for multiple extended targets in temporally correlated cognitive radar system," IET Radar, Sonar \& Navigation, vol. 10, pp. 398-410, 2016.
[17] W. Yue, L. Li, Y. Xin et al., "Radar constant-modulus waveform optimization for high-resolution range profiling of stationary targets," Sensors, vol. 17, no. 11, p. 2574, 2017.

[18] H.-S. Kim, N. A. Goodman, C. K. Lee, and S.-I. Yang, "Improved waveform design for radar target classification," Electronics Letters, vol. 53, no. 13, pp. 879-881, 2017.

[19] W. Yue, Y. Zhang, and J. Xie, "Radar constant-modulus waveform design for multiple extended targets," IEICE Transactions on Fundamentals of Electronics, Communications and Computer Sciences, vol. 100, no. 3, pp. 888-892, 2017.

[20] L. Wang, W. Zhu, Y. Zhang, Q. Liao, and J. Tang, "Multitarget detection and adaptive waveform design for cognitive MIMO radar," IEEE Sensors Journal, vol. 18, no. 24, pp. 9962-9970, 2018.

[21] T. Hao, C. Cui, and Y. Gong, "Efficient low-PAR waveform design method for extended target estimation based on information theory in cognitive radar," Entropy, vol. 21, no. 3, p. 261, 2019.

[22] X. Cheng, D. A. Aubry, and X. A. Wangde Maio, "Robust waveform and filter bank design of polarimetric radar," IEEE Transactions on Aerospace and Electronic Systems, vol. 53, no. 1, pp. 370-384, 2017.

[23] Y. Yang, Z. Blum, and D. R. Fuhrmann, "MIMO radar waveform design via alternating projection," IEEE Transactions on Signal Processing, vol. 58, no. 3, pp. 1440-1445, 2010.

[24] D. Ciuonzo, G. A. De Maio, and M. Piezzo, "Intrapulse radarembedded communications via multiobjective optimization," IEEE Transactions on Aerospace and Electronic Systems, vol. 51, no. 4, pp. 2960-2974, 2015. 